\title{
Análise comparativa entre índices de vegetação NDVI e EVI e sua relação com o balanço hídrico na Bacia do Una - SP
}

\author{
Yhasmin Mendes de Moura ${ }^{1}$ \\ Yosio Edemir Shimabukuro ${ }^{2}$ \\ Gustavo Bayma Siqueira da Silva ${ }^{2}$ \\ Gilberto Fisch ${ }^{1}$ \\ Nelson Wellausen Dias ${ }^{1}$ \\ Diogo Gadioli ${ }^{1}$ \\ ${ }^{1}$ Universidade de Taubaté - UNITAU \\ Caixa Postal 12.020-040 - Taubaté - SP - Brasil
}

yhasmin@dsr.inpe.br; gfisch@iae.cta.br ; nelson.dias@unitau.br; digadioli@hotmail.com

\author{
${ }^{2}$ Instituto Nacional de Pesquisas Espaciais - INPE \\ Caixa Postal 515 - 12245-970 - São José dos Campos - SP, Brasil \\ \{yosio, bayma\}@dsr.inpe.br
}

\begin{abstract}
The objective of this work was to evaluate the interannual behavior of the main land cover classes (Agriculture, Forest and Capoeira, and Pasture) that composes the Una river hydrographic basin, located in Taubaté, São Paulo state. For this, the vegetation index (IV) data from MOD13 product, NDVI (Normalized Difference Vegetation Index) and EVI (Enhanced Vegetation Index), and the water balance data of the basin were used. The results showed that the IVs followed the seasonality of the region (highest in the rainy season and lowest during the dry season). The correlation between IVs and evapotranspiration was highest for EVI in the Agriculture and Pasture classes (Pearson= 0, 72 and 0, 56, respectively). We concluded that EVI obtained satisfactory results to differentiate the behavior of the vegetation classes in the analysis of meteorology parameters influence in the region.
\end{abstract}

Palavras-chave: remote sensing, evapotranspiration, MODIS, sensoriamento remoto, evapotranspiração, MODIS.

\section{Introdução}

Alguns estudos sobre fenologia vegetal utilizam, como dados auxiliares, informações referentes à variabilidade hídrica do solo, as características morfológicas da cobertura vegetal que compõem a paisagem, e até mesmo o nível de degradação decorrente de certas atividades antrópicas. As bacias hidrográficas são vulneráveis às alterações da cobertura vegetal, pois estas interferem nas propriedades dos solos. Isto é refletido nas propriedades da água dos rios, influenciando em suas características bioquímicas e no ciclo hidrológico do manancial, que é de fundamental importância para a sustentabilidade da bacia (Balbinot et al., 2008).

Desta forma, a quantidade de água armazenada no solo acaba por influenciar os processos atmosféricos, hidrológicos e biológicos, influenciando a previsão do tempo e a quantificação de estresses hídricos da cobertura vegetal durante os períodos de estiagem.

Nesse contexto, é necessário um melhor entendimento da interligação das variações fenológicas com os diferentes componentes do meio geográfico (clima, solo, relevo, geologia, etc). As mudanças nos padrões de diversos tipos de formações vegetais, como no período de crescimento, na taxa de acumulação de biomassa e na taxa de senescência, podem ser fortemente influenciadas pela temperatura e precipitação (Anderson \& Shimabukuro, 2007). De acordo com Metzger (1999), o processo antrópico de fragmentação modifica a estrutura da paisagem, resultando em mudanças na composição e na diversidade das formações vegetais. 
Estudos apresentam resultados favoráveis e demonstram a potencialidade no acompanhamento da dinâmica de Uso e Cobertura da Terra (UCT), permitindo representações de avaliação dos estágios fenológicos e fisiológicos da cobertura florestal (Gurgel \& Ferreira, 2003; Huete et al., 2006; Lee et al., 2003; Varriale \& Gomes, 2001; White et al., 2005). A estrutura e produtividade do ecossistema são sensíveis às mudanças no balanço hídrico, que podem ser causados pelas mudanças meteorológicas (Scholes \& Breemen, 1997).

Além disso, Houborg et al. (2007) destacaram que essas estimativas, ao definirem o status da vegetação, constituem importantes inputs em modelos que quantificam a troca de energia e matéria entre a superfície terrestre e a atmosfera. Para compreensão desta dinâmica nos estudos florestais, a utilização de produtos e técnicas de extração de informações derivadas de dados obtidos remotamente vem se mostrando útil, devido ao avanço tecnológico. Esta ferramenta tem demonstrado potencial no acompanhamento da dinâmica de UCT e seus possíveis impactos, através do uso de Índices de Vegetação (que são formulações derivadas de modelos lineares ou não de bandas espectrais), que permite a avaliação das condições fenológicas da cobertura florestal, em nível regional ou global (Lee et al., 2003).

A utilização de técnicas de sensoriamento remoto (SR) no monitoramento da resposta fenológica da vegetação podem auxiliar na compreensão de fenômenos ecológicos, como também servir de subsídio ao planejamento sustentável da cobertura florestal. Além disso, podem contribuir para prognósticos sobre o crescimento e produtividade florestal, facilitando a implantação de programas de manejo, utilização racional e recuperação da cobertura florestal (Carey et al., 1994; Hatshorn, 1990).

Percebe-se que nos últimos anos ocorreu aumento de estudos relacionados às variáveis ambientais e a detecção de mudanças fenológicas através da utilização das imagens MODIS (Gurgel \& Ferreira, 2003; Li et al., 2001; Ribeiro et al., 2007).

Neste sentido, este trabalho tem como objetivo analisar o comportamento interanual de tipologias vegetais através do Índice de Vegetação da Diferença Normalizada (NDVI) e do Índice de Vegetação Melhorado (EVI) do sensor MODIS e sua relação com o balanço hídrico na bacia do Una - SP.

\section{Materiais e Métodos}

A bacia hidrográfica do Una está localizada entre os paralelos $23^{\circ} 05^{\prime}$ e $22^{\circ} 40^{\prime} \mathrm{S}$ e $45^{\circ}$ $42^{\prime}$ e $45^{\circ} 22^{\prime} \mathrm{W}$ (Figura 1), ocupando aproximadamente 47.676 ha. Está situada no médio Vale do Paraíba e abrange parte dos municípios de Taubaté, Tremembé, Pindamonhangaba e Redenção da Serra, situados no cone leste do Estado de São Paulo (Batista et al., 2005).

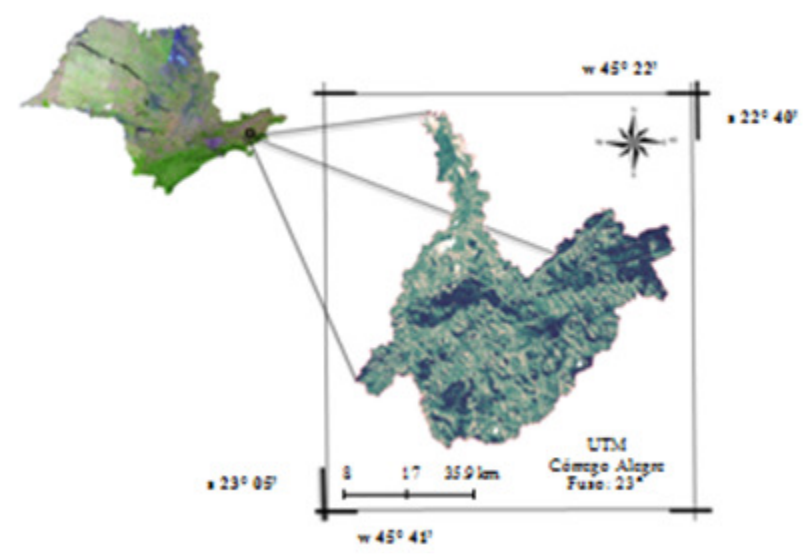

Figura 1 - Localização da bacia do Rio Una. 
Com base nos dados de UCT oriundos do Projeto Una (Batista et al., 2005) foram separadas as classes "Agricultura", "Mata ou Capoeira", "Pasto" e "Reflorestamento" que correspondem às coberturas vegetais predominantes na bacia. Ainda, foi realizada a união das subdivisões Pasto Degradado e Pasto Sujo à classe Pasto e o mesmo para a classe de Reflorestamento, que foi unida à classe de Reflorestamento Cortado, formando apenas uma classe representativa destas feições. De acordo com o Projeto Una as quatro classes são especificadas como (i) Agricultura: áreas que representam cobertura do solo com uso agrícola, culturas anuais ou perenes; (ii) Mata ou Capoeira: áreas de vegetação natural primária, pioneira ou secundária em estágio inicial, médio ou avançado de regeneração; (iii) Pasto: áreas ocupadas por pastagens naturais ou implantadas, áreas que apresentam erosão, e ainda áreas que apresentam quadro de abandono na utilização e manutenção, permitindo a evolução de estágios de regeneração da vegetação; e (iv) Reflorestamento: áreas ocupadas por reflorestamento com Eucaliptus sp., Pinus sp. , ou outras espécies exóticas que sofreram ou não corte raso na extração de madeira (Figura 2).
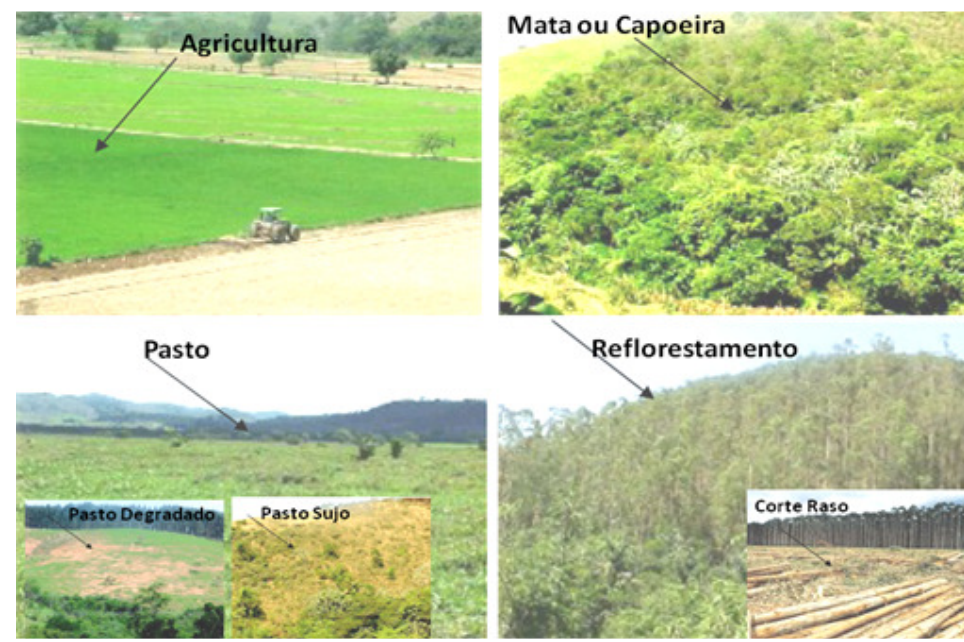

Figura 2 - Classes de vegetação utilizadas: Agricultura, Mata ou Capoeira, Pasto e Reflorestamento.

Fonte: adaptada de Batista et al. (2005).

Para cada classe foram definidas 30 amostras espalhadas sobre a área, escolhidas de forma que não houvesse sobreposição dos pixels entre cada classe, permitindo desta forma representar melhor o comportamento de cada classe evitando a mistura.

Dos dados do produto MOD13 foram utilizadas 12 datas, cada uma correspondendo a um mês do ano de 2008. Este ano apresentou-se dentro das características climatológicas da região de Taubaté. O NDVI é um índice mais sensível à clorofila e o EVI possui maior sensibilidade às variações na resposta estrutural do dossel, incluindo o índice de área foliar (LAI), a fisionomia da planta e a arquitetura do dossel (Huete et al., 2008). Estes índices de vegetação são obtidos a partir das seguintes equações:

Onde:

$$
N D V I=\frac{\rho_{\mathrm{IVP}}-\rho_{\mathrm{V}}}{\rho_{I V P}+\rho_{V}}
$$

$\mathbf{I V P}=$ reflectância do infravermelho próximo;

$\mathbf{\rho V}=$ reflectância do vermelho. 
Onde:

$$
\mathrm{EVI}=\mathrm{G} * \frac{\rho_{\mathrm{IVP}}-\rho_{\mathrm{V}}}{\rho_{\mathrm{IVP}}+\mathrm{C}_{1} * \rho_{\mathrm{V}}-\mathrm{C}_{2} * \rho_{\mathrm{A}}+\mathrm{L}}
$$

IIVP = reflectância do infravermelho próximo;

$\boldsymbol{\rho} \mathbf{V}=$ reflectância do vermelho;

$\mathbf{\rho A}=$ reflectância do azul;

$\boldsymbol{C 1}$ = coeficiente de ajuste para efeito de aerossóis da atmosfera no vermelho;

$\boldsymbol{C 2}$ = coeficiente de ajuste para efeito de aerossóis da atmosfera no azul;

$\mathbf{L}=$ fator de ajuste para o solo;

$\mathbf{G}=$ fator de ganho.

Os dados climáticos utilizados para o cálculo do balanço hídrico foram coletados no posto meteorológico do Departamento de Ciências Agrárias da Universidade de Taubaté (UNITAU), localizado no município de Taubaté, na região do Vale do Rio Paraíba do Sul. Este posto está situado nas coordenadas geográficas $23^{\circ} 02^{\prime} \mathrm{S}$ e $45^{\circ} 30^{\prime} \mathrm{W}$, em uma cota de 577 metros acima do nível do mar. Os dados de evapotranspiração foram calculados segundo o método Penman-Monteith, método padrão utilizado pela FAO (Organização das Nações Unidas para Agricultura e Alimentação), que utiliza os dados climáticos de radiação solar, vento, temperatura do ar e déficit de pressão de vapor. A partir destes dados foram realizadas análises estatísticas das médias e desvios padrões dos IVs das classes de UCT, e posterior correlação (coeficiente de Pearson) entre os dados de evapotranspiração e IVs para o ano de 2008.

\section{Resultados e Discussão}

O balanço hídrico possibilita estimar além da evapotranspiração potencial, a evapotranspiração real, o excedente hídrico, a deficiência hídrica e as fases de reposição e retirada de água do solo (capacidade de armazenamento, com nível máximo adotado de 100mm). Pode-se observar na Figura 3 (b) que os maiores excedentes ocorrem na estação chuvosa, e os déficits nos meses de estação seca. Verifica-se também uma estabilidade hídrica durante os meses de transição (maio e junho).
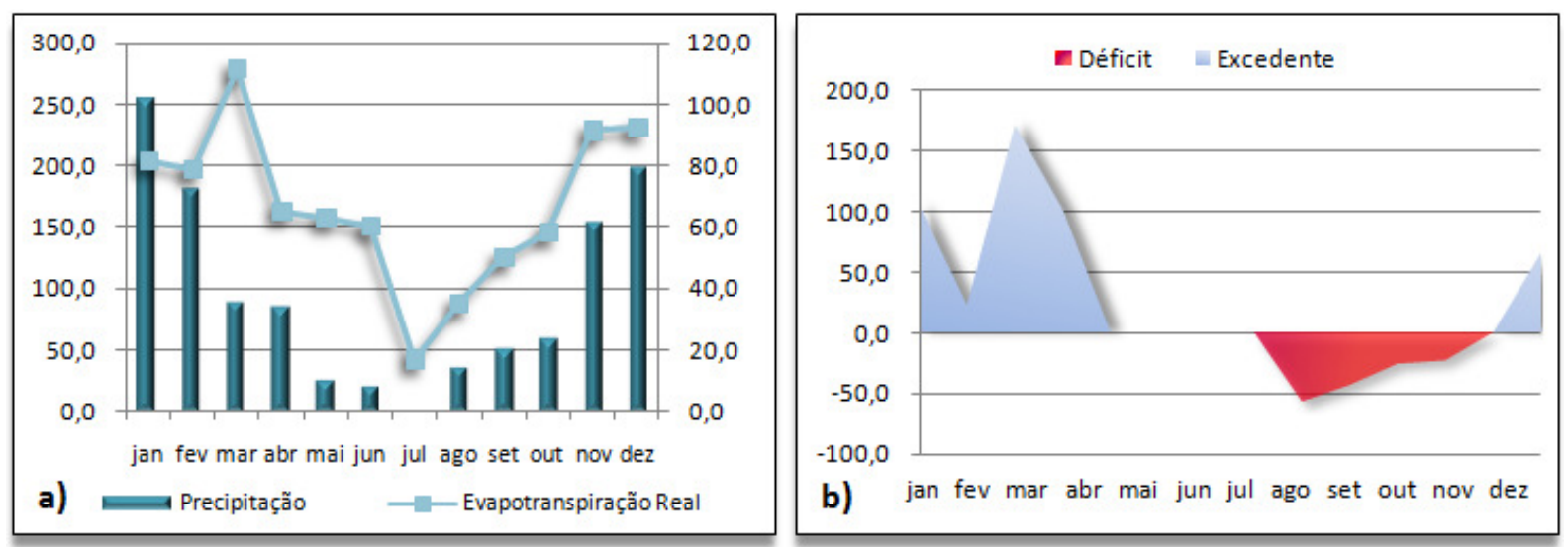

Figura 3 - a) Precipitação e evapotranspiração (mm/mês); b) Balanço Hídrico do município de Taubaté - SP, com armazenamento de água no solo de $100 \mathrm{~mm}$, para o ano de 2008.

A Figura 4 apresenta o comportamento do EVI e NDVI nas classes de UCT para o ano de 2008. Em termos gerais verificam-se, para os IVs, que todas as classes apresentaram dois 
períodos de valores distintos, maiores no período chuvoso (dez., jan., fev. e mar.) e menores no período seco (jun., jul. e ago.).

Verificou-se que o comportamento dos IVs da classe Agricultura acompanhou a sazonalidade da região, e obtiveram valores mais altos nos meses de estação chuvosa (dez., jan., fev., mar), tanto para o EVI quanto para o NDVI. No entanto, o EVI apresentou maior sensibilidade às restrições hídricas nos meses de maio, junho e julho, e também apresentou um comportamento ascendente no início da estação chuvosa da região, que se inicia a partir de setembro (Folhes \& Fisch, 2006), a agricultura vai estar associada também aos períodos de irrigação interferindo, portanto, na oferta de água para esta classe.

A classe Mata ou Capoeira apresentou um comportamento sazonal diferente para os dois índices, sendo que os maiores valores no período chuvoso no EVI, enquanto o NDVI não apresentou uma variação brusca nos períodos de estação seca e chuvosa, salvo o mês de setembro. Pode ser indicada a variação do EVI no mês de julho, quando a disponibilidade de água é menor na região. No entanto, para estas formações florestais a disponibilidade hídrica propicia uma estabilidade mesmo na época seca, devido à atividade fotossintética e conseqüente produção de fitomassa foliar mantidas nesta estação (Huete et al., 2006). Os valores mais baixos para os meses de maio, junho e agosto podem estar relacionados à queda da fitomassa foliar, a qual depende da disponibilidade hídrica atingida pelas raízes mais profundas.

A classe Pasto apresentou um comportamento mais associado à sazonalidade da região através do NDVI. Este apresentou, de modo geral, maiores valores nos meses da estação chuvosa e os menores na seca. Para ambos os índices houve um aumento no valor no mês de julho, isto pode estar associado à mudança na cobertura vegetal da área, pois os dados de delimitação das classes são de 2003 e os índices de vegetação de 2008, podendo ter ocorrido um aumento das áreas de pasto sujo com regeneração da vegetação nativa de um estágio inicial para um estágio mais avançado.

Na classe Reflorestamento, o EVI apresentou um comportamento mais associado à variação sazonal da região do que o NDVI. A região é marcada pela presença de reflorestamento por eucalipto, neste caso, no final da estação seca, fluxos ascendentes do lençol freático para superfície ajudam a manter o sistema florestal em funcionamento. Isto pode ter ocorrido para o mês de setembro na classe reflorestamento, diminuindo as restrições sazonais de água para esta cultura. Porém, ocorreu diminuição do valor no mês de outubro, o que pode ser decorrente do déficit hídrico que ocorre até o mês de novembro, demonstrando que neste período o reflorestamento pode estar diminuindo sua capacidade de captação de água no solo.
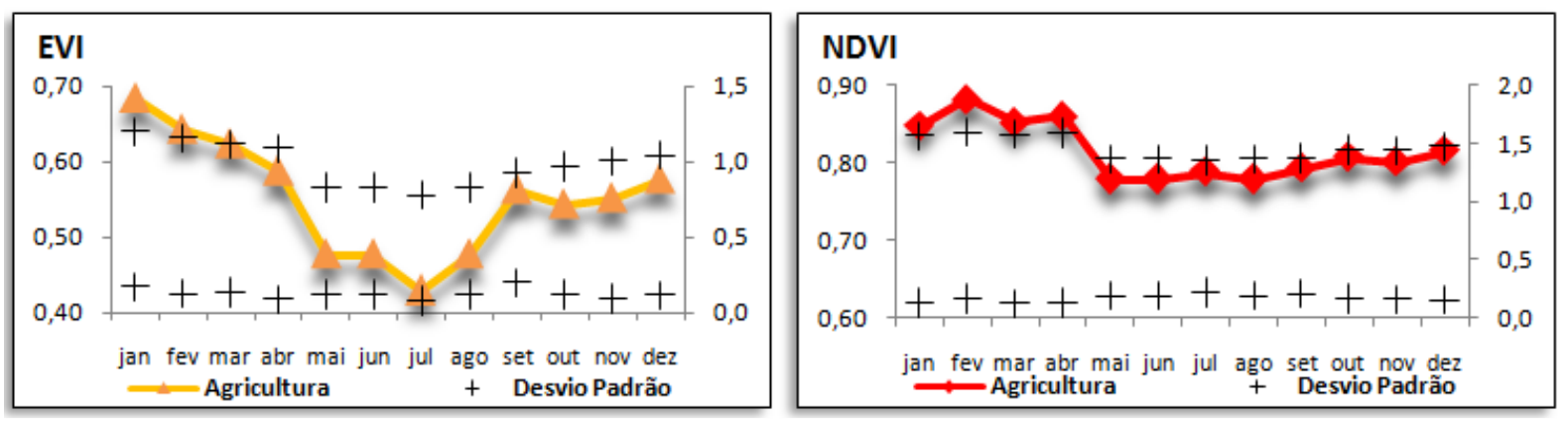

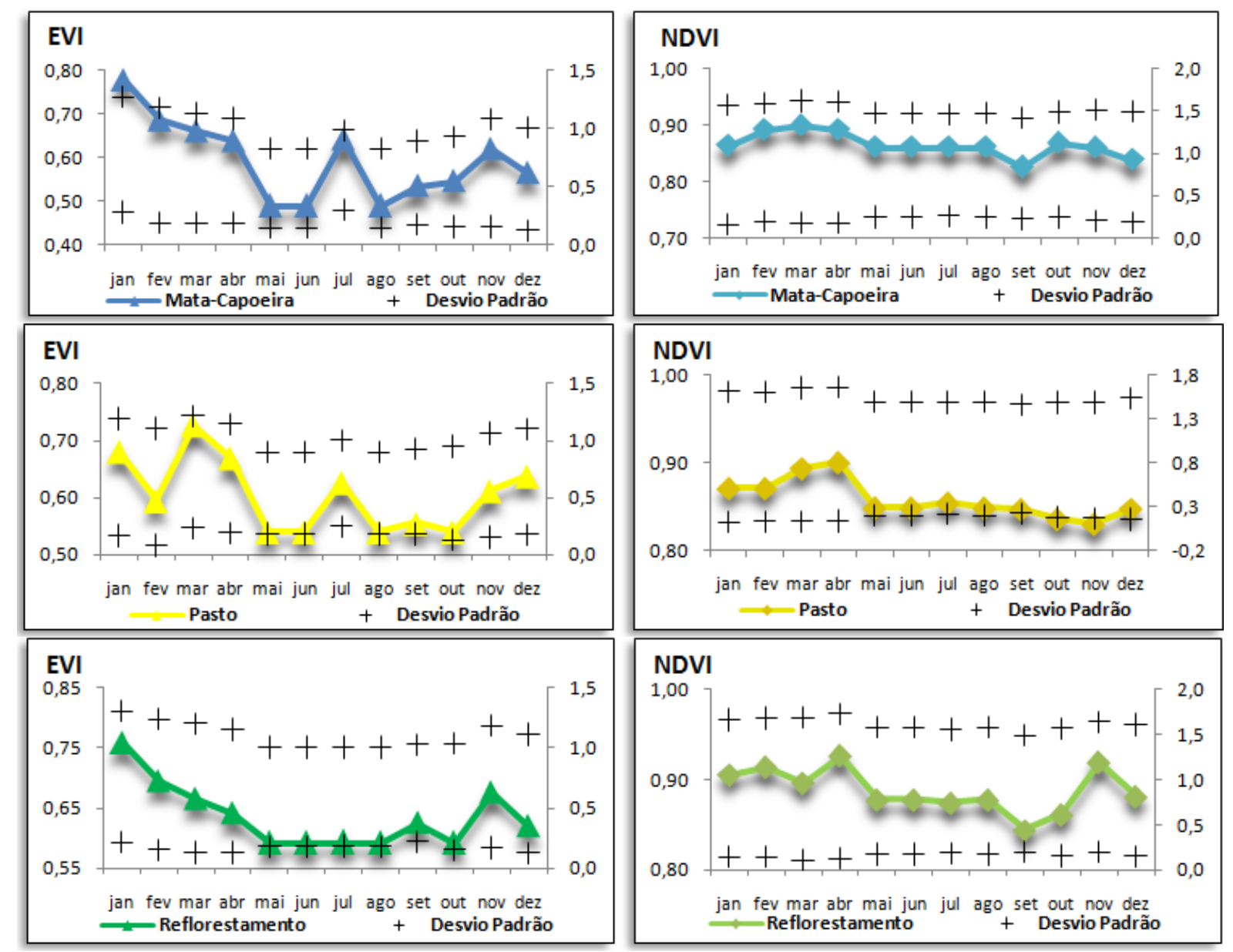

Figura 4 - Comportamento mensal-anual do EVI e NDVI das classes Agricultura, MataCapoeira, Pasto e Reflorestamento na Bacia do Una - SP.

A Tabela 2 apresenta a correlação de cada uma das classes Agricultura, Mata-Capoeira, Pasto e Reflorestamento para o EVI e NDVI para o ano de 2008.

Tabela 2 - Correlação (Pearson) entre EVI, NDVI e Evapotranspiração na Bacia do Una - SP.

\begin{tabular}{c|c|c|c|c}
\cline { 2 - 5 } & Agricultura & Mata ou Capoeira & Pasto & Reflorestamento \\
\hline NDVI & 0,55 & 0,32 & 0,30 & 0,50 \\
\hline EVI & 0,72 & 0,38 & 0,56 & 0,58 \\
\hline
\end{tabular}

Pode-se dizer que em termos gerais o EVI apresentou maior correlação com a evapotranspiração para as classes de UCT na bacia do Rio Una, principalmente para as classes Agricultura e Pasto. Isto pode estar associado à maior sensibilidade por parte destas fisionomias às variações hídricas da região. Também pode ser observado que para as classes Mata ou Capoeira e Reflorestamento as correlações são moderadas, indicando por parte destas fisionomias uma menor dependência das variações hídricas.

\section{Conclusões}

A inserção de dados referentes ao balanço hídrico, na análise do comportamento sazonal da vegetação na Bacia do Rio Una, permitiu a compreensão mais adequada da dinâmica sazonal dos diferentes tipos de cobertura vegetal para o ano de 2008. Pode-se verificar que 
houve uma maior eficácia por parte do EVI para avaliação do comportamento das classes, evidenciado principalmente nas classes Mata ou Capoeira e Reflorestamento, nas análises sazonais, e Agricultura e Pasto, nas análises de correlação. Desta forma, o trabalho corrobora para o tratamento metodológico nas análises da influência dos parâmetros meteorológicos no comportamento fenológico das principais classes de UCT da região.

\section{Referências}

Anderson, L. O. et al. Monitoramento da resposta fenológica através da utilização de imagens fração derivadas do sensor MODIS. In: Simpósio de Sensoriamento Remoto (SBSR), 12., 2005, Goiânia. Anais... São José dos Campos: INPE, 2005. Artigos, p. 2811-2818, 2005. CD-ROM, On-line. Disponível em: $<$ http://marte.dpi.inpe.br/rep-/ltid.inpe.br/ sbsr/2004>. Acesso em: 05 ago. 2009.

Anderson, L. O.; Shimabukuro, Y. E. Classificação espectro-temporal de formações vegetais. In: Rudorff, B. F. T.; Shimabukuro, Y. E.; Ceballos, J. C. (Org.). O sensor MODIS e suas aplicações no Brasil. São José dos Campos: Editora Parêntese, cap. 20, p.277-294, 2007.

Balbinot, R.; de Oliveira, N. K.; Vanzetto, S. C.; Pedroso, K.; Valerio, A. F. O papel da floresta no ciclo hidrológico em bacias hidrográficas. Ambiência, v.4 n.1 p.131-149, 2008.

Batista, G. T.; Targa, M. S.; Fidalgo, E. C. C. Banco de dados ambientais da Bacia do Rio Una, Bacia do Rio Paraíba do Sul. DSPACE. (http://www.agro.unitau.br:8080/ dspace/handle/2315/51), Repositório Eletrônico Ciências Agrárias, Coleção Ciências Ambientais. 16p., 2005.

Carey, E.; Brown, S.; Gillespie, A. J. R.; Lugo, A. Tree mortality in mature lowland tropical moist forest and tropical lower mountain moist forests of Venezuela. Biotropica, v. 26, p. 255-265, 1994.

Folhes, M. T.; Fisch, G., Caracterização climática e estudo de tendências nas séries temporais de temperatura do ar e precipitação em Taubaté (SP). Revista Ambi-Água, Taubaté, v. 1, n. 1, p. 61-71, 2006.

Gurgel, H. C.; Ferreira, N. J. Annual and interannual variability of NDVI in Brazil and its connections with climate. International Journal of Remote Sensing, v.24, n. 18, p. 3595-3609, 2003.

Hartshorn, G. S. An overview of neotropical forest dynamics. p. 585-600 In: Gentry, A. H. Four neotropical rainforests. Yale University Press, New Haven, p. 585 - 600, 1990.

Houborg, R., Soegaard, H., Boegh, E. Combining vegetation index and model inversion methods for the extraction of key vegetation biophysical parameters using Terra and Aqua MODIS reflectance data. Remote Sensing of Environment, v.106, n.1, p.39-58, 2007.

Huete, A. et al. MODIS vegetation index (MOD13) algorithm theoretical basis document. versão 3, p. 1-129, 1999. Disponível em: <modis-land.gsfc.nasa.gov/pdfs/atbd_mod13.pdf>. Acesso em: 10 set. 2009.

Huete, A. R.; Kamel, D.; Shimabukuro, Y. E.; Ratana, P.; Saleska, S. R.; Hutyra, L. R.; Yang, W.; Nemani, R. R.; Myneni, R. Amazon rainforests green-up with sunlight in dry season. Geophysical Research Letters, v. 33, N. 106405, P. 1-4, 2006.

Lee, R.; Yu, F.; Price, K. P.; Ellis, J.; Shi, P. Evaluating vegetation phenological patterns in winner Mongolia using NDVI time-series analysis. International Journal of Remote Sensing, v.23, n.12, p. $2505-2512,2007$.

Trishchenko A. et al. Effects of spectral response function on surface reflectance and NDVI measured with moderate resolution satellite sensors. Remote Sensing of Environment, v. 81, p. 1 - 18. 2002.

Metzger, J. P. Estrutura da paisagem e fragmentação: uma análise bibliográfica. Anais da Academia Brasileira de Ciências, v. 71, p. 445 - 463, 1999. 
Ribeiro, C. A. D. et al. Banco de dados temporal de imagens NDVI do sensor MODIS para o Espírito Santo. In: Simpósio Brasileiro de Sensoriamento Remoto (SBSR), 13, 2007, Florianópolis. Anais... São José dos Campos: INPE, 2007. Artigos, p. 6075-6081, 2007. CD-ROM, On-line. Disponível em: <http://marte.dpi.inpe.br/rep/ltid.inpe.br/sbsr/2007>. Acesso em: 22 ago. 2009.

Scholes R.J.; Breemen N. The effects of global change on tropical ecosystems. Geoderma, v. 79, p. 9-24, 1997.

Varriale, M. C.; Gomes, A. G. Modelagem de ecossistemas uma introdução - Santa Maria, RS: UFSM, p. 503, 2001.

White, M. A.; Hoffman, F.; Hargrove, W. W.; Nemani, R. R. A global framework for monitoring phenological responses to climate change. Geophysical Research Letters, v. 32, n. L04705, p.1-4, 2005. 